\title{
Electron Capture Dissociation at Low Temperatures Reveals Selective Dissociations
}

\author{
Romulus Mihalca, Anne J. Kleinnijenhuis, ${ }^{*}$ and Liam A. McDonnell \\ FOM Institute for Atomic and Molecular Physics (AMOLF), Amsterdam, The Netherlands
}

\author{
Albert J. R. Heck and Ron M. A. Heerent \\ Department of Biomolecular Mass Spectrometry, Bijvoet Center for Biomolecular Research, Utrecht Institute \\ for Pharmaceutical Sciences, Utrecht University, Utrecht, The Netherlands
}

\begin{abstract}
Electron capture dissociation at $86 \mathrm{~K}$ of the linear peptide Substance $\mathrm{P}$ produced just two backbone fragments, whereas at room temperature eight backbone fragments were formed. Similarly, with the cyclic peptide gramicidin S, just one backbone fragment was formed at $86 \mathrm{~K}$ but five at room temperature. The observation that some backbone scissions are active and others inactive, when all involve $\mathrm{N}-\mathrm{C}_{\alpha}$ cleavages and have a high rate constant, indicates that the more specific fragments at low temperatures reflects the reduced conformation heterogeneity at low temperatures. This is supported by reduced or inactive hydrogen loss, a channel that has previously been shown to be affected by conformation. The conclusion that the ECD fragments are a snapshot of the conformational (intramolecular solvation shell) heterogeneity helps explain how the relative intensities of ECD fragments can be different on different instrument and highlights the common theme in methodologies used to increase sequence coverage, namely an increase in the conformational heterogeneity of the precursor ion population. (J Am Soc Mass Spectrom 2004, 15, 1869-1873) (C 2004 American Society for Mass Spectrometry
\end{abstract}

$\mathrm{E}$ lectron capture dissociation (ECD) [1-4] employs reactions of multiprotonated peptides/ proteins with low energy electrons to generate peptide fragments. The high sequence coverage and the ability to retain labile groups after ECD have allowed posttranslational modifications (PTMs) and point mutations (PMs) in peptides and proteins to be both identified and localized. Such performance is of great value for the analysis of the proteome, particularly that of diseased organisms. PTMs and PMs are widespread and are frequently associated with disease [5-7], for example over 80 different point mutations have been found in transthyretin (most of which lead to autosomal disorders) [8].

To be able to fully characterize such modified proteins, $100 \%$ sequence coverage is required (so-called top-down proteomics). Several methods have been developed to increase the sequence coverage of ECD, which can be grouped into either infrared illumination [9-11] or collisional activation [12]. These methods increase the average internal energy of the ions, which also affects the conformation of these gas-phase ions [13].

Published online November 19, 2004

Address reprint requests to Professor R. M. A. Heeren, FOM Institute for Atomic and Molecular Physics, Kruislaan 407, 1098 SJ Amsterdam, The Netherlands. E-mail: heeren@amolf.nl

† Also at the FOM Institute for Atomic and Molecular Physics (AMOLF) Amsterdam, The Netherlands.
There is experimental and theoretical evidence that ECD is directed by the internal solvation of the (neutralized) proton $[9,14-18]$. If the reaction progresses faster than the electron-proton recombination energy is randomized and thermal fluctuations are small, specific fragments would be expected from a single (frozen) conformer, reflecting the solvation shell, specific for that conformation, surrounding the neutralized proton. Thermal fluctuations that result in a dynamic solvation shell, but not conformational change (local fluctuations rather than a global change), would produce a greater number of fragments. Finally, multiple conformations as well as thermal fluctuations would produce yet more fragments. Because the methods used to increase the sequence coverage of ECD all increase the internal energy of the ions, and so affect the conformation of the gas-phase ions, the greater sequence coverage could simply reflect the greater conformational heterogeneity (and "thermal" motion) of the activated ion population. To investigate the importance of conformational heterogeneity on the fragments produced by ECD, we have performed ECD at $86 \mathrm{~K}$. If the extent of fragmentation is governed by the conformational heterogeneity, less but more specific fragments will be obtained at $86 \mathrm{~K}$.

Here we report significant changes in ECD spectra at low temperature compared with those at near room temperature, performed on a linear and a cyclic peptide under otherwise identical conditions. 


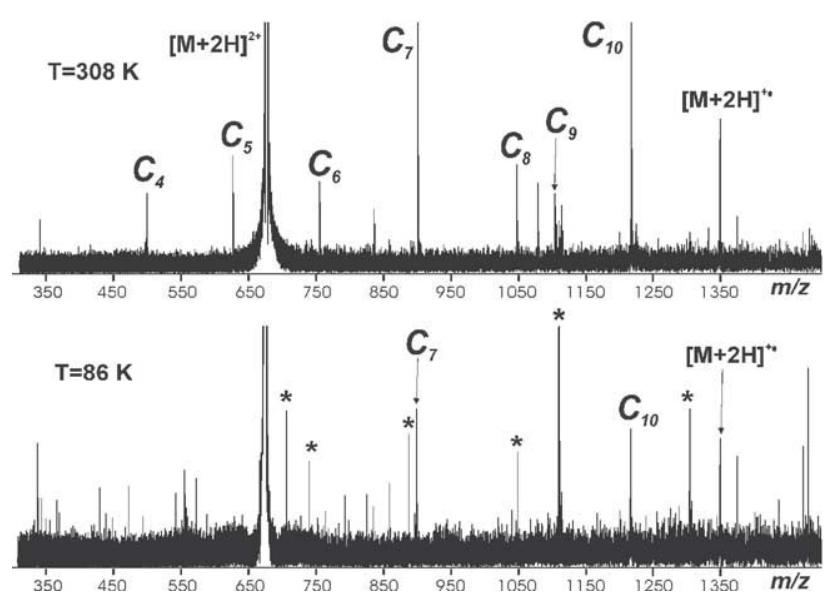

Figure 1. Comparison of the ECD spectra of the $[\mathrm{M}+2 \mathrm{H}]^{2+}$ ion of Substance P at $308 \mathrm{~K}(\mathbf{a})$ and $86 \mathrm{~K}(\mathbf{b})$. Both spectra are the average of 50 scans.

\section{Experimental}

Gas phase ions of the linear peptide Substance $P$ (RPKPQQFFGLM) and the cyclic peptide gramicidin $\mathrm{S}$ (VOLFPVOLFP) were generated using electrospray ionization $(20 \mu \mathrm{M}$ in $1: 1$ water:acetonitrile $+1 \%$ formic acid) and injected into a Fourier transform ion cyclotron resonance mass spectrometer (FTMS) equipped with an electron gun and a temperaturecontrolled cell [19]. Following isolation of the [M + $2 \mathrm{H}]^{2+}$ ions using a stored waveform inverse Fourier transform pulse [20], the precursor ions were left to equilibrate at the requested temperature for $50 \mathrm{~s}$ before undergoing a $400 \mathrm{~ms}$ ECD event. To investigate the temperature dependence of ECD, experiments were performed on ion-populations thermalized near room temperature and at $86 \mathrm{~K}$. These represent the two temperatures under which most ECD experiments are performed and an extremely low temperature (the lowest temperature amenable to our experimental set-up) that would detect any temperature dependence.

\section{Results and Discussion}

Substance $\mathrm{P}$ was chosen because it is a linear peptide that under normal conditions (room temperature) produces many backbone fragments. ECD of Substance P at $308 \mathrm{~K}$ produced seven $c$ fragments $\left(c_{4-10}\right)$ and a weak $z_{9}$. fragment, in agreement with previous studies [21]. Under otherwise identical conditions, except performing the experiment at $86 \mathrm{~K}$, the $c_{4}, c_{5}, c_{6}, c_{8} . c_{9}$, and $z_{9}$. fragments were not detected. Only the $c_{7}$ and $c_{10}$ fragments were observed at $86 \mathrm{~K}$. Figure 1 shows a comparison of these two spectra, the disappearance of six of the eight fragments is readily apparent.

Figure 2 shows three representative examples in more detail, the $c_{5}$ and $c_{9}$ fragments that both disappeared at $86 \mathrm{~K}$ and the $c_{10}$ fragments that remained. The top row shows the fragment spectra at $308 \mathrm{~K}$ and the bottom the analogous spectra at $86 \mathrm{~K}$. It is clear that a smaller number of fragments were detected at $86 \mathrm{~K}$.

ECD is thought to occur close to the charge-reduced protonation sites through internal solvation of the proton and $\mathrm{H}$ transfer after electron capture [2, 16-18]. (Note: it was recently suggested that electron capture could occur prior to proton transfer [22], the results shown here cannot distinguish between these two possibilities). The fragments formed by these fast mechanisms would be expected to be sensitive to the conformation of the molecule and, extrapolating, the fragments formed by an ion population would be sensitive to the conformational heterogeneity of the ion packet. At higher temperatures (or after collisional/ radiative activation) the number of conformations and the thermal fluctuations are larger. Consequently, more fragments are expected at higher temperatures (internal energies). Figure 1 shows that the fragments obtained after ECD of Substance P at $86 \mathrm{~K}$ and $308 \mathrm{~K}$ fit this scenario, namely more fragments are obtained at higher temperatures because of the larger conformational space occupied by the molecule. The sensitivity of the fragments formed by ECD to the conformation population also explains why the relative abundances of the $c$ fragments have differed on different instruments. The following relative intensities have all been reported $c_{5}>$ $c_{4}>c_{6}>c_{7}>c_{8}>c_{9}>c_{10}$ [23], $c_{5}>c_{6}>c_{7}>c_{4}>$ $c_{9}>c_{10}>c_{8}$ [11], and $c_{10}>c_{7}>c_{9}>c_{8}>c_{6}>c_{5}>>$ $c_{4}$ [24]. The different fragment intensities could reflect the different conformation populations present. The conformation population is influenced by the final internal energy distribution of the ions, which can be altered during ionization, ion transport through the mass spectrometer, and ion trapping in the ICR cell (where ECD occurs). These parameters are almost certainly different for experiments performed on different instruments operated by different people.

A common reaction pathway found in ECD experiments is loss of a hydrogen atom (the reduced proton).

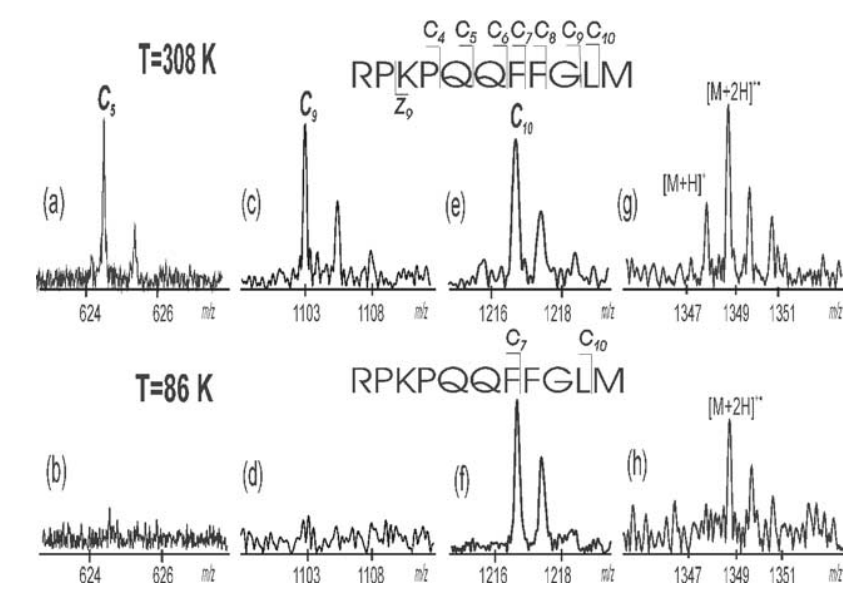

Figure 2. $\quad c_{5}$ fragment of Substance P after ECD at (a) 308 and (b) $86 \mathrm{~K} ; c_{9}$ fragment of Substance P after ECD at (c) 308 and (d) $86 \mathrm{~K}$; $c_{10}$ fragment of Substance P after ECD at (e) 308 and (f) $86 \mathrm{~K}$; [M $+\mathrm{H}]^{+}$and $[\mathrm{M}+2 \mathrm{H}]^{+\cdot}$ cluster at (g) 308 and (h) $86 \mathrm{~K}$. 
Different degrees of hydrogen loss have been attributed to conformational change [25]. In support of the above conformation explanation for the different fragments detected at 308 and $86 \mathrm{~K}$, it was found that the hydrogen loss channel was active at the higher temperature but not at the lower temperature (Figure 2). All of the above observations are consistent with the premise that the reaction pathways followed after electron capture, and thus the fragments obtained, reflect the conformational heterogeneity of the trapped ions. It is encouraging that the fragments obtained at $86 \mathrm{~K}$ appear to correlate with the structure calculated by Bowers and workers for the gas-phase $[\mathrm{M}+2 \mathrm{H}]^{2+}$ ion of Substance $\mathrm{P}$, in which the part of the peptide backbone involved in internal solvation includes the amino acids whose scission leads to the $c_{7}$ and $c_{10}$ fragments [26]. Intramolecular solvation of the protonated lysine is $\approx 40 \mathrm{~kJ} \mathrm{~mol}^{-1}$ more stable (enthalpically) than solvation of the other protonation site (arginine) and the enthalpic contribution increasingly dominates the conformer population at lower temperatures (see personal communications below) (and thus the intramolecular solvation shells that determine which fragments are obtained).

The ability to cleave $\mathrm{N}-\mathrm{C}_{\alpha}$ bonds but retain labile groups after ECD and the time-scales of other recombination-dissociation reactions lead to the postulate that ECD is a non-ergodic process, that dissociation occurs prior to energy randomization [2]. However, more recent studies have concluded that, for these radical reactions, nonergodicity does not need to be invoked because of the low activation barriers of the backbone cleavages [27]. For Substance P the total internal energy after ECD is 3.9 (4.6) and $6.2(6.9) \mathrm{eV}$, for neutralization of the lysine (arginine) protonation site at 86 and $308 \mathrm{~K}$, respectively. These internal energies are sufficient for unimolecular rate constants $>10^{5} \mathrm{~s}^{-1}$ when distributed throughout the molecule [27]. The question as to whether the smaller number of fragments at $86 \mathrm{~K}$ reflects the lower conformational heterogeneity before energy randomization or after requires further study to determine the energies associated with the conformational changes that lead to different fragments. Future plans include performing temperature resolved ion mobility studies to determine the internal energies associated with these conformational changes.

ECD of gramicidin S (VOLFPVOLFP) at $86 \mathrm{~K}$ also showed differences from that performed near room temperature. At $313 \mathrm{~K}$ three types of fragments were detected: small molecule losses, five backbone cleavages (labeled B1-5 in Figure 3) and side chain losses, in agreement with previous studies [28]. At $86 \mathrm{~K}$ a smaller number of each type of fragment was detected, for example just one backbone fragment (labeled B1 in Figure 3). Because gramicidin S is cyclic, these backbone fragments require two cleavages from a single electroncapture event and are thought to arise from a freeradical cascade [28]. While it is pertinent to contemplate whether the reduced number of fragments is attributable to the radical cascade terminating earlier at the lower internal energies at $86 \mathrm{~K}$, considering the low activation barriers of these gas-phase radical reactions $[18,27]$ it is not sufficient without invoking a conformational argument. As with the Substance P results, the smaller number of fragments is consistent with a smaller conformer population and reduced thermal fluctuations. This is also supported by the observation that the hydrogen loss channel was significantly attenuated at $86 \mathrm{~K}$ (Figure 3).

Recently, Iavarone et al. reported that the number of $c$ fragments obtained from ECD of the synthetic peptide AcHN-(AKAAK) ${ }_{3} \mathrm{~A}-\mathrm{NH}_{2}$ was insensitive to temperature from room temperature up to $150{ }^{\circ} \mathrm{C}$. This was interpreted as indicating that the products are "... not due to effects of tertiary structure" [29]. This behavior is in stark contrast to the cold temperature results reported here and several previous studies. Breuker et al. have reported increased sequence coverage of ubiquitin by heating throughout the same temperature range [9], Håkansson et al. and Tsybin et al. have increased the number of fragments by heating the ion cloud with an infrared laser [10, 11], Horn et al. have increased the sequence coverage by using collisional activation to heat the ion cloud [30], and in addition to temperature dependent ECD, Adams et al. found that a single D-amino acid substitution, which is known to affect solution-phase secondary and tertiary structure, significantly affected the ECD fragments obtained [16].

The different behavior seen by Iavarone reflects the unusual nature of the peptide used in their study. This peptide has previously been shown to exhibit an unusually stable helix, especially in non-polar solvents (high vacuum is also non-polar) [31]. Increased helix formation has been associated with a smaller number of ECD fragments because the carbonyl groups that would otherwise solvate the (neutralized) proton and lead to $\mathrm{N}-\mathrm{C}_{\alpha}$ scission are involved in the hydrogen-bond network that stabilizes the helix. Consequently, it is the unusual stability of the helix in this peptide that is

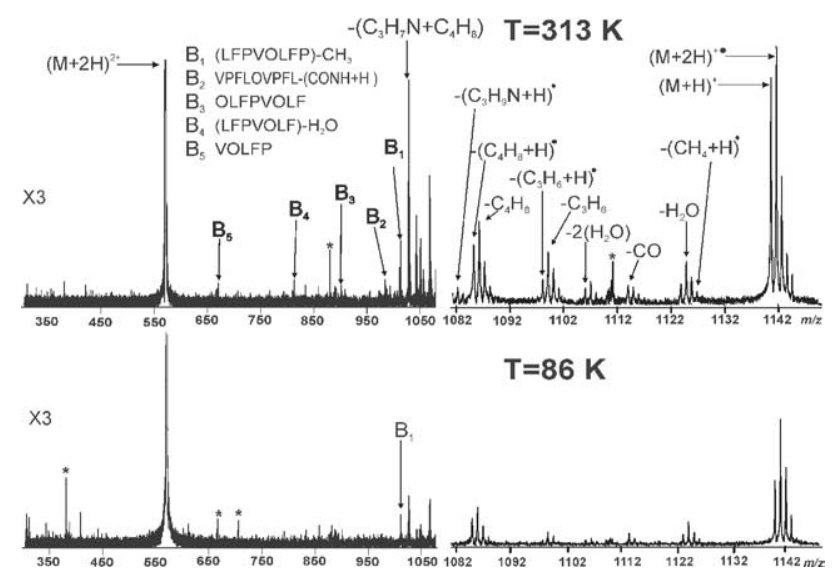

Figure 3. A comparison of gramicidin S ECD fragments at 313 and $86 \mathrm{~K}$. The backbone fragments have been labeled B1 through $\mathrm{B} 5$, their respective sequences have been indicated in the figure legend. Both spectra are the average of 25 scans. 
probably responsible for the limited number of ECD fragments throughout the temperature range investigated. In this regard, the results of Iavarone et al. would provide further support for the crucial role of conformation in ECD.

All of the results discussed above demonstrate that the fragments produced by ECD of multiprotonated peptides/proteins are significantly influenced by the conformations of the gas phase ions because this determines which groups are involved in the intramolecular solvation of the (neutralized) proton. The results presented here show that at $86 \mathrm{~K}$ ECD generates much fewer fragments than at room temperature, for both Substance P and gramicidin S. This reflects the reduced conformational heterogeneity at lower temperatures, not the least because the low activation barriers associated with gas-phase radical reactions makes any argument based on reaction rates problematic. Such conformational arguments are consistent with observations of increased sequence coverage using techniques that heat the ion population and provide an explanation why the relative intensities of ECD fragments can be different on different instruments. For increased sequence coverage these results indicate that it is advisable to heat (energize) the ion population in order to obtain a heterogeneous conformation population. When analyzing biomolecules that contain labile post-translational modifications, this should be performed with due care in order to ensure that these labile groups are retained. Such modifications are associated with many diseases and ECD provides an exceptional methodology for identifying and localizing these modifications. With sufficient care a balance can be reached in which the ion population is energized enough for extensive sequence information but the labile modifications are retained $[10,12,32]$.

\section{Conclusions}

Electron capture dissociation at $86 \mathrm{~K}$ of the linear peptide Substance $\mathrm{P}$ and the cyclic peptide gramicidin $\mathrm{S}$ generated significantly fewer fragments than at room temperature. It is inferred that these more specific fragments reflect the conformational heterogeneity of the ion population because the reactions are still fast at $86 \mathrm{~K}\left(>10^{5} \mathrm{~s}^{-1}\right.$ for the common ECD channels) and directed by intramolecular hydrogen bonds, and the similarity of the channels for fragments that were active and inactive make any alternative explanation implausible. At higher temperatures, a greater number of conformations as well as larger thermal fluctuations leads to more fragments. Changes to the degree of hydrogen loss, a reaction pathway previously shown to be influenced by conformation, supports this assertion. Moreover, the specific Substance P fragments at $86 \mathrm{~K}$ are consistent with preferential intramolecular solvation of the lysine.

The results presented here indicate how the conformation (solvation shell) population of the ion packet affect the relative intensities of the fragments and sequence coverage. This last aspect is important because the most attractive feature of ECD is its ability to identify and localize post-translational modifications to proteins, which are associated with many of the diseases of present day concern (Alzheimer's, Parkinson's, and cancer).

\section{Acknowledgments}

The authors acknowledge generous funding from the Stichting voor Fundamenteel Onderzoek der Materie (FOM), which is financially supported by the Nederlandse organisatie voor Wetenschappelijk Onderzoek (NWO). They thank Professor Dr. František Tureček for calculations and helpful comments, and Marc Duursma for technical assistance.

\section{Addendum}

In a personal communication in 2004, Turecek, F. writes as follows. "B3-PMP2/6-311 ++ G(2d,p) calculations of ionization energies give $\mathrm{IE}_{\mathrm{a}}=3.00 \mathrm{eV}$ for the $\varepsilon$-ammonium radical from $\mathrm{N}_{\alpha}$-acetyl-lysine amide and $\mathrm{IE}_{\mathrm{a}}=$ $3.53 \mathrm{eV}$ for the guanidinium radical from $\mathrm{N}_{\alpha}$-acetylarginine amide. The vertical recombination energies are somewhat lower, $\mathrm{RE}_{\mathrm{v}}=2.84$ and $2.44 \mathrm{eV}$ for the lysine and arginine cations, respectively. The coulomb contribution to recombination energy in doubly charged RPKPKQQFFGLM has been calculated from fully optimized structures (by PM3) of RPK-NH2 motifs combined with Mulliken and NPA charge distributions with the B3LYP / 6-31G* wave function. The more stable conformer with internally solvated Lys side chain has $\mathrm{E}_{\text {coulomb }}=0.60-0.73 \mathrm{eV}$ (NPA and Mulliken populations, respectively). The less stable conformer $(\mathrm{DE}=$ $+24 \mathrm{~kJ} \mathrm{~mol}^{-1}$ ) with internally solvated Arg side chain has $\mathrm{E}_{\text {coulomb }}=0.85 \mathrm{eV}$. Thus, the total recombination energy in a Lys-solvated [RPKPKQQFFGLM $+2 \mathrm{H}]^{2+}$ conformer should be close to $3.00+0.7=3.7 \mathrm{eV}$ for electron capture at the Lys residue and $3.53+0.7=4.23$ $\mathrm{eV}$ for electron capture at the Arg residue. Using previous data for peptide heat capacities, [RPKPKQQFFGLM $+2 \mathrm{H}]^{2+}$ is estimated to have 0.2 and 2.5 eV internal energy $\left(H_{\mathrm{T}}\right)$ at 86 and $308 \mathrm{~K}$, respectively. Upon adding the electron recombination energy, the "cold" Lys-reduced ion will have a total of $0.2+3.7=$ $3.9 \mathrm{eV}$ internal energy, corresponding to an effective temperature of $385 \mathrm{~K}$. The "ambient" ion will have 2.5 $+3.7=6.2 \mathrm{eV}$, corresponding to $495 \mathrm{~K}$. The Argreduced ions will be slightly more energetic, 4.6 and 6.9 $\mathrm{eV}$ for the "cold" and "ambient" systems, corresponding to 415 and $520 \mathrm{~K}$, respectively."

\section{References}

1. Zubarev, R. A.; Kruger, N. A.; Fridriksson, E. K.; Lewis, M. A.; Horn, D. M.; Carpenter, B. K.; McLafferty, F. W. Electron Capture Dissociation of Gaseous Multiply-Charged Proteins is Favored at Disulfide Bonds and Other Sites of High Hydrogen Atom Affinity. J. Am. Chem. Soc. 1999, 121, 2857-2862. 
2. Zubarev, R. A. Reactions of Polypetide Ions with Electrons in the Gas Phase. Mass Spectrom. Rev. 2003, 22, 57-77.

3. Zubarev, R. A.; Horn, D. M.; Fredricksson, E. K.; Kelleher, N. L.; Kruger, N. A.; Lewis, M. A.; Carpenter, B. K.; McLafferty, F. W. Electron Capture Dissociation for Structural Characterization of Multiply Charged Protein Cations. Anal. Chem. 2000, 72, 563-573.

4. Zubarev, R. A.; Kelleher, N. L.; McLafferty, F. W. Electron Capture Dissociation of Multiply Charged Protein Cations. A Nonergodic Process. J. Am. Chem. Soc. 1998, 120, 3265-3266.

5. Ge, Y.; ElNaggar, M.; Sze, S. K.; Oh, H. B.; Begley, T. P.; McLafferty, F. W.; Boshoff, H.; Barry, C. E. III. Top Down Characterization of Secreted Proteins from Mycobacterium tuberculosis by Electron Capture Dissociation Mass Spectrometry. J. Am. Soc. Mass Spectrom. 2003, 14, 253-261.

6. Hanash, S. Disease Proteomics. Nature 2003, 422, 226-232.

7. Pierson, J.; Norris, J. I.; Aerni, H.-R.; Svenningsson, P.; Caprioli, R. M.; Andrén, P. E. Molecular Profiling of Experimental Parkinson's Disease: Direct Analysis of Peptides and Proteins on Brain Tissue Sections by MALDI Mass Spectrometry. J. Proteome Res. 2004, 3, 289-295.

8. Connors, L. H.; Richardson, A. M.; Théberge, R.; Costello, C. E. Tabulation of Transthyretin (TTR) Variants as of $1 / 1 / 2000$. Amyloid-Journal Protein Folding Disorder 2000, 7, 54-69.

9. Breuker, K.; Oh, H.; Horn, D. M.; Cerda, B. A.; McLafferty, F. W. Detailed Unfolding and Folding of Gaseous Ubiquitin Ions Characterized by Electron Capture Dissociation. J. Am. Chem. Soc. 2002, 124, 6407-6420.

10. Håkansson, K.; Chalmers, M. J.; Quinn, J. P.; McFarland, M. A.; Hendrickson, C. L.; Marshall, A. G. Combined Electron Capture and Infrared Multiphoton Dissociation for Multistage MS/MS in a Fourier Transform Ion Cyclotron Resonance Mass Spectrometer. Anal. Chem. 2003, 75, 3256-3262.

11. Tsybin, Y. O.; Witt, M.; Baykut, G.; Kjeldsen, F.; Håkansson, P. Combined Infrared Multiphoton Dissociation and Electron Capture Dissociation with a Hollow Electron Beam in Fourier Transform Ion Cyclotron Resonance Mass Spectrometry. Rapid Comm. Mass Spectrom. 2003, 17, 1759-1768.

12. Sze, S. K.; Ge, Y.; Oh, H.; McLafferty, F. W. Top-Down Mass Spectrometry of a 29-kDa Protein for Characterization of Any Posttranslational Modification to Within One Residue. PNAS 2002, 99, 1774-1779.

13. Jarrold, M. F. Peptides and Proteins in the Vapor Phase. Annu. Rev. Phys. Chem. 2000, 51, 179-207.

14. Oh, H.-B.; Breuker, K.; Sze, S. K.; Ge, Y.; Carpenter, B. K.; McLafferty, F. W. Secondary and Tertiary Structures of Gaseous Protein Ions Characterized by Electron Capture Dissociation Mass Spectrometry and Photofragment Spectroscopy. PNAS 2002, 99, 15863-15868.

15. Fung, Y. M. E., Duan, L., Chan, T.-W. D. Top-Down Characterization of Secreted Proteins from Mycobacterium Tuberculosis by Electron Capture Dissociation Mass Spectrometry. Eur. J. Mass Spectrom. 2004, in press.

16. Adams, C. M.; Kjeldsen, F.; Zubarev, R. A.; Budnik, B. A.; Haselmann, K. F. Electron Capture Dissociation Distinguishes a Single D-Amino Acid in a Protein and Probes Tertiary Structure. J. Am. Soc. Mass Spectrom. 2004, 15, 1087-1098.

17. Haselmann, K. F.; Budnik, B. A.; Kjeldsen, F.; Polfer, N. C.; Zubarev, R. A. Can the (M--X) Region in Electron Capture Dissociation Provide Reliable Information on Amino Acid
Composition of Polypeptides. Eur. J. Mass Spectrom. 2002, 8 461-469.

18. Turecek, F.; Syrstad, E. A.; Seymour, J. L.; Chen, X.; Yao, C. Peptide cation-radicals. A Computational Study of the Competition Between Peptide N-Ca Bond Cleavage and Loss of the Side Chain in the [GlyPhe-NH2 $+2 \mathrm{H}]+$. Cation-Radical. J. Mass Spectrom. 2003, 38, 1093-1104.

19. Guo, X.; Duursma, M.; Al-Khalili, A.; McDonnell, L. A.; Heeren, R. M. A. Design and Performance of a New FT-ICR Cell Operating at a Temperature Range of 77-438 K. Int. J. Mass Spectrom. 2004, 231, 37-45.

20. Marshall, A. G.; Wang, T. C. L.; Ricca, T. L. Tailored Excitation for Fourier Transform Ion Cyclotron Mass Spectrometry. J. Am. Chem. Soc. 1985, 107, 7893-7897.

21. Axelsson, J.; Palmblad, M.; Håkansson, K.; Håkansson, P. Electron Capture Dissociation of Substance P Using a Commercially Available Fourier Transform Ion Cyclotron Resonance Mass Spectrometer. Rapid Commun. Mass Spectrom. 1999, 13, 474-477.

22. Syrstad, E. A.; Turecek, F. Computational Evidence for Direct Electron Attachment to the Peptide Backbone in ECD. Proceedings of the 52nd ASMS Conference on Mass Spectrometry; Nashville, TN, 2004;

23. Chan, T.-W. D.; Ip, W. H. H. Optimization of Experimental Parameters for Electron Capture Dissociation of Peptides in a Fourier Transform Mass Spectrometer. J. Am. Soc. Mass Spectrom. 2002, 13, 1396-1406.

24. McFarland, M. A.; Hudgins, R. R.; Håakansson, K.; Hendrickson, C. L.; Marshall, A. G. ECD of peptides following gas-phase H/D exchange, Proceedings of the 50th ASMS Conference on Mass Spectrometry and Allied Topics; Orlando, FL, 2002.

25. Breuker, K.; Oh, H.; Cerda, B. A.; Horn, D. M.; McLafferty, F. W. Hydrogen Atom Loss in Electron-Capture Dissociation: A Fourier Transform-Ion Cyclotron Resonance Study with Single Isotopomeric Ubiquitin Ions. Eur. J. Mass Spectrom. 2002, 8, 177-180.

26. Gill, A. C.; Jennings, K. R.; Wyttenbach, T.; Bowers, M. T. Conformations of Biopolymers in the Gas Phase: A New Mass Spectrometric Method. Int. J. Mass Spectrom. 2000, 195/196, 685-697.

27. Turecek, F. N-C $\alpha$ Bond Dissociation Energies and Kinetics in Amide and Peptide Radicals. Is the Dissociation a NonErgodic Process? J. Am. Chem. Soc. 2003, 125, 5954-5963.

28. Leymarie, N.; Costello, C. E.; O'Connor, P. B. Electron Capture Dissociation Initiates a Free Radical Reaction Cascade. J. Am. Chem. Soc. 2003, 125, 8949-8958.

29. Iavarone, A. T.; Paech, K.; Williams, E. R. Effects of Charge State and Cationizing Agent on the Electron Capture Dissociation of a Peptide. Anal. Chem. 2004, 76, 2231-2238.

30. Horn, D. M.; Ge, Y.; McLafferty, F. W. Activated Ion Electron Capture Dissociation for Mass Spectral Sequencing of Larger (42 kDa) Proteins. Anal. Chem. 2000, 72, 4778-4784.

31. Marqusee, S.; Robbins, V. H.; Baldwin, R. L. Unusually Stable Helix Formation in Short-Alanine Based Peptides. PNAS 1989, 86, 5286-5290.

32. Chalmers, M. J.; Håkansson, K.; Johnson, R.; Smith, R.; Shen, J.; Emmett, M. R.; Marshall, A. G. Protein Kinase A Phosphorylation Characterized by Tandem Fourier Transform Ion Cyclotron Resonance Mass Spectrometry. Proteomics 2004, 4, 970981. 